\title{
Numerical Simulation for Flow Field in a Sump
}

\author{
Prakash Shinde ${ }^{1}$, Dr. V. N. Raibhole ${ }^{2}$, Ajinkya Satam ${ }^{3}$ \\ ${ }^{1}$ (Kirloskar Systech Ltd., India) \\ ${ }^{2}$ (Department of Mechanical Engineering, MES College of Engineering, India) \\ ${ }^{3}$ (Department of Mechanical Engineering, K.E. Society's Polytechnic, India)
}

\begin{abstract}
The efficiency and performance of pumping stations involving multiple pumping units depends not only on the efficiency of the pumping units but also on the proper design of the Intake sump. Proper design of pump intake is not an easy task because of the various site-specific geometrical and hydraulic constraints. The time and cost involved in sump model studies for design of sump geometry can be reduced to a large extent through CFD studies as we can predict the flow parameters at the pump inlet with the change in geometry without actual running of pump with CFD. Hence the design of sump can be optimized to keep the flow parameters below limiting values. In this thesis, an attempt was made to minimize the swirl angles and increase the flow at the pump inlet.
\end{abstract}

Keywords - Intake Hydraulic, Pump Inlet, Swirl Angle, Sump Design, Vortex, Guide Wall.

\section{INTRODUCTION}

Generally CFD analysis is carried out where there is a flow of water or any stream involving sumps, pumps, pipe/channels etc. The fluid flow in sumps is rather complex involving expansions, turns together with fluid structure interaction. It is essential to ensure that pumps operating in such sumps get smooth swirl free flow at its entry. Traditional approach was to carry out sump model studies experimentally with a reduced scale model and applying Froude Similarity rules. Computational Fluid Dynamics (CFD) has recently come up as an alternative approach to investigate the complex fluid flow phenomenon in sumps. It is a software based application to study various fluid dynamics. It is rapidly becoming an important tool for analysis and design in hydraulic engineering. Hydraulic engineering encompasses a broad range of activity from flow in a river to design of structures to control and distribute/divert water for various purposes. These flow problems have features that are not commonly found in other applications. In the case of pump industry applications, CFD tools are important in view of analysis of the hydraulic passage of the pumps and sumps. In case of sumps, CFD analysis is used to investigate flow quality entering into the pump at various combinations of pumps in operation. The analysis is done at minimum water level with pumps running at duty point.

\section{OBJECTIVE}

To carry out the detailed sump flow analysis using CFD tool and modify the sump geometry to bring out the optimized flow parameters for the smooth functioning of a pump.

\section{LITERATURE REVIEW}

Journal of Experimental Methods in Marine Hydrodynamics [1], was referred to use General Modelling and Scaling Laws for scaling down the domain by maintaining the dynamic similarity. Froude's model was used for scaling of domain. Samir A. S. Ibrahim [2], tested the design of the pump intake and investigated modifications on the design which enhanced the efficiency of the pump intake structure with the help of HIS recommendations Kadam Pratap M. etal [3], developed CFD model which was used to study the effect of various parameters which reduces time as well as cost and hence can become an important tool for optimization of pump sump geometry. CFD results were validated with the experimental data including swirl angle. Swirl angles were significantly reduced and flow was uniformly distributed. V.K Gahlot etal [4], predicted the three dimensional flow and vortices in a pump sump model. The CFD model predicted the flow pattern in detail and the location, and nature of the vortices. Thus it was concluded that CFD model can be used to study the effect of various parameters and hence can become an important tool for optimization of pump sump geometry. Tanweer S. Desmukh etal [5], confirmed that the flow through the pump sump is greatly affected by the geometry of sump and reducing the angle of expansion in the horizontal plane improves the flow conditions considerably. American National Standards for Pump Intake Design [6] recommended the standard sump design modification methods which are being widely used for optimizing the sump design to reduce the swirl angles and improve the flow velocity at the intake of pump. CHEN Hong-xun etal [7], presented numerical model for three-dimensional 
turbulent flow in the sump of the pump station. A reasonable boundary condition for the flow in the sump with multi-intakes, each of which may have different flow rates has been proposed. Shazy A. Shabayek [8], developed an original model by installation of side walls and curtain walls for the lateral distribution of flow within circulating water pump bays and to reduce the vortex activity in the vicinity of the pump such that the hydraulic conditions in the sump would meet the specified performance criteria.

\section{METHODOLOGY}

Table 1 - Methodology Details

\begin{tabular}{|l|l|}
\hline Modelling: & ProEngineer Wildfire 5.0 \\
\hline Package Used & ANSA v14.0.2 \\
\hline Meshing: & Tetrahedral \\
\hline Package Used & Fluent 14.5 \\
\hline Mesh Type & 3D \\
\hline Solver: & RANS, Viscous standard k-e \\
\hline Package Used & \multicolumn{2}{|l|}{} \\
\hline Simulation & CFD-Post 14.5 \\
\hline Turbulent Model
\end{tabular}

\section{MODEL R0:}

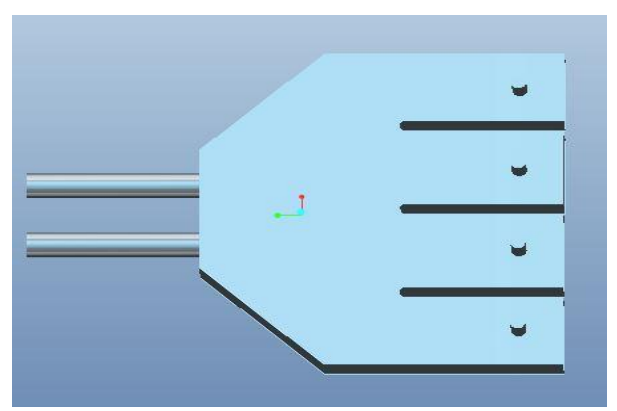

Fig.1 CAD Model R0

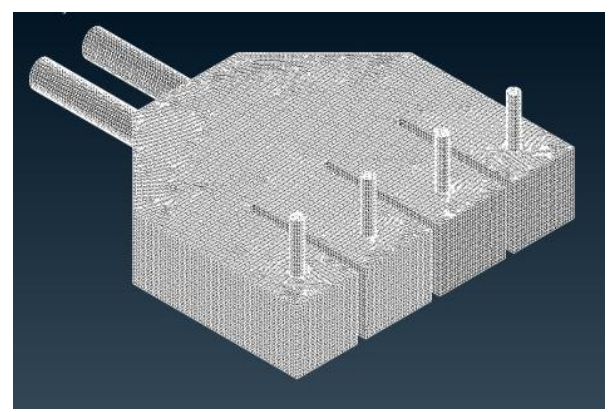

Fig.2 Meshed Model R0

CAD modelling was done with a ProEngineer Wildfire 5.0 and grid generation was done with ANSA 14.0.4. Volumetric meshing with unstructured tetra meshing option was adopted [2], [3], [4], [5].

Table 2 - Grid Independence Test

\begin{tabular}{|c|c|c|c|c|c|}
\hline GRID & No. of Nodes & No. of Elements & $\begin{array}{c}\text { Swirl Angle in } \\
\text { Tube 1 }\end{array}$ & $\begin{array}{c}\text { Experimental } \\
\text { Value }\end{array}$ & Error \\
\hline G1 & 222241 & 1.2 million & 7 & 7.41 & $5.53 \%$ \\
\hline G2 & 236739 & 1.26 million & 7.214 & 7.41 & $2.65 \%$ \\
\hline G3 & 248026 & 1.34 million & 7.215 & 7.41 & $2.63 \%$ \\
\hline
\end{tabular}

It can be clearly seen that values obtained from G2 and G3 are almost same. Even though in G3 mesh count is larger, result has changed with a negligible amount. Hence G2 was selected and the results presented in this paper are all simulated using G2 grid.

Table 3- Design Modification Table:

\begin{tabular}{|c|c|c|c|}
\hline BELL DIA & RELATION & R0 & R1 \\
\hline \multirow{4}{*}{$0.136 \mathrm{~m}$} & $\mathrm{X} \geq 5 \mathrm{D} \geq 0.68 \mathrm{~m}$ & $0.775 \mathrm{~m}$ & $0.7 \mathrm{~m}$ \\
\cline { 2 - 4 } & $\mathrm{Z}_{1} \geq 5 \mathrm{D} \geq 0.68 \mathrm{~m}$ & $1.266 \mathrm{~m}$ & $0.7 \mathrm{~m}$ \\
\cline { 2 - 4 } & $\mathrm{W}=2 \mathrm{D}=0.272 \mathrm{~m}$ & $0.575 \mathrm{~m}$ & $0.3 \mathrm{~m}$ \\
\cline { 2 - 4 } & $\mathrm{B}=0.75 \mathrm{D}=0.102 \mathrm{~m}$ & $0.287 \mathrm{~m}$ & $0.1 \mathrm{~m}$ \\
\cline { 2 - 4 } & $\mathbf{C}=\mathbf{0 . 5 5 D} \square \mathbf{0 . 0 7 5 m}$ & $0.075 \mathrm{~m}$ & $0.075 \mathrm{~m}$ \\
\hline
\end{tabular}

Other modifications:

1. Chamfer with dimension of 0.5D in pump bay to direct the flow towards bell entrance 
2. Domain is divided into two parts with a gate opening for maintenance purpose MODEL R1:

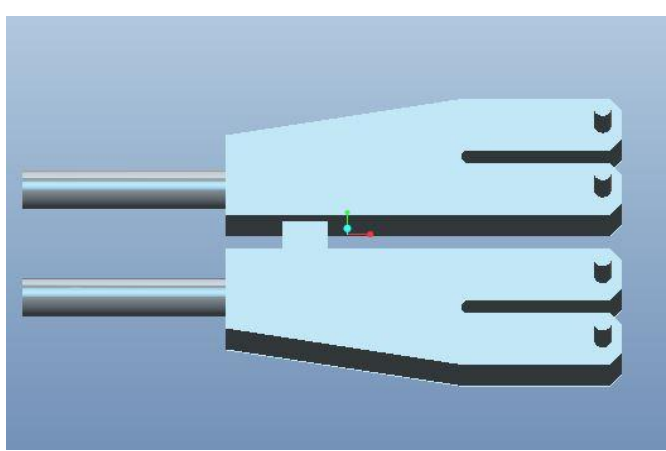

Fig.3 Solid Model R1

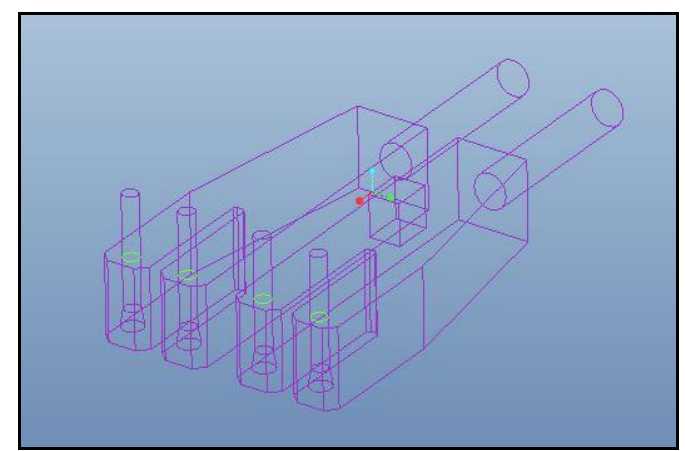

Fig.4 Wireframe Model R1

\section{CFD RESULTS}

\section{STREAMLINE PLOTS:}

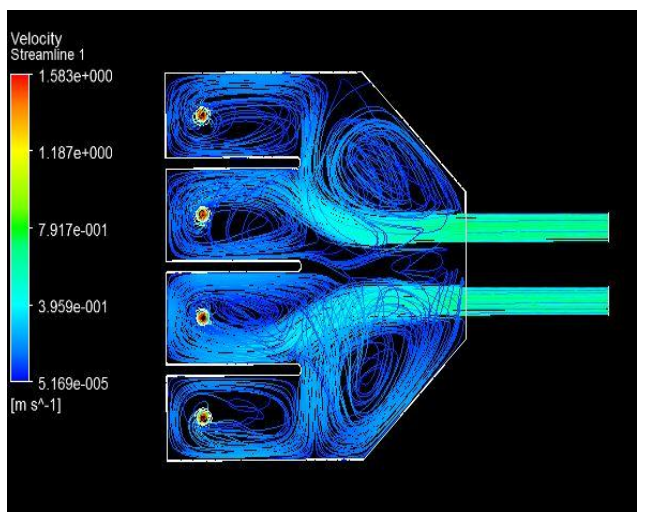

Fig.5 R0

\section{VECTOR PLOTS:}

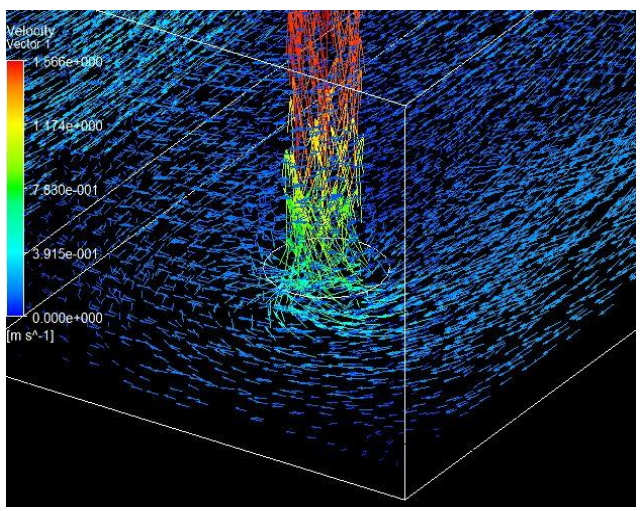

Fig.7 R0

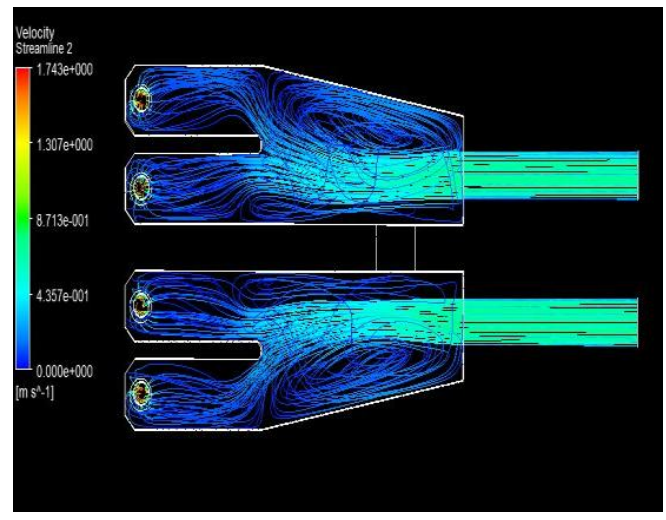

Fig.6 R1

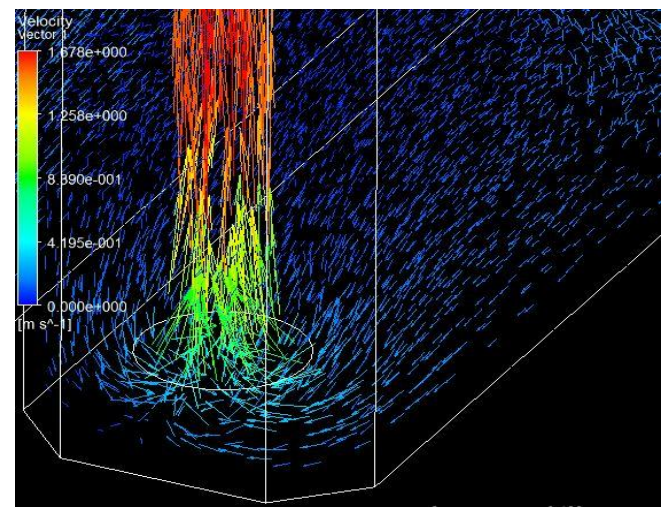

Fig.8 R1

From streamline plots we can conclude that the flow pattern has been smoothen and in vector plot we can observe that particles of fluid are directed towards the bell mouth in model R1 rather than scattering or reflecting back in model $\mathrm{R} 0$. 
VELOCITY CONTOURS:

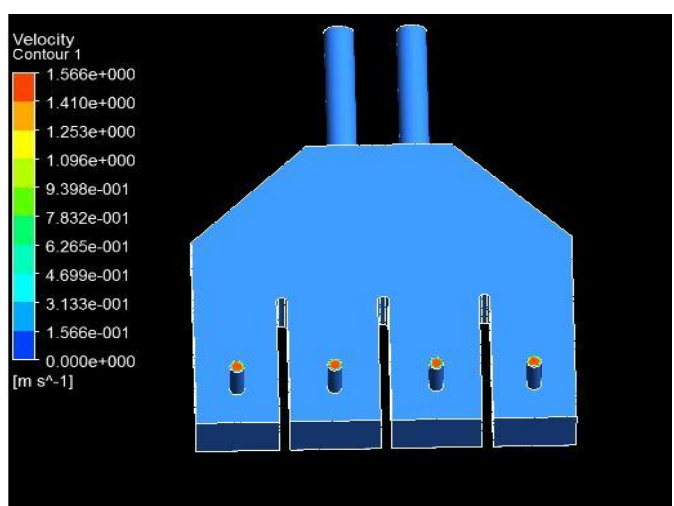

Fig.9 R0

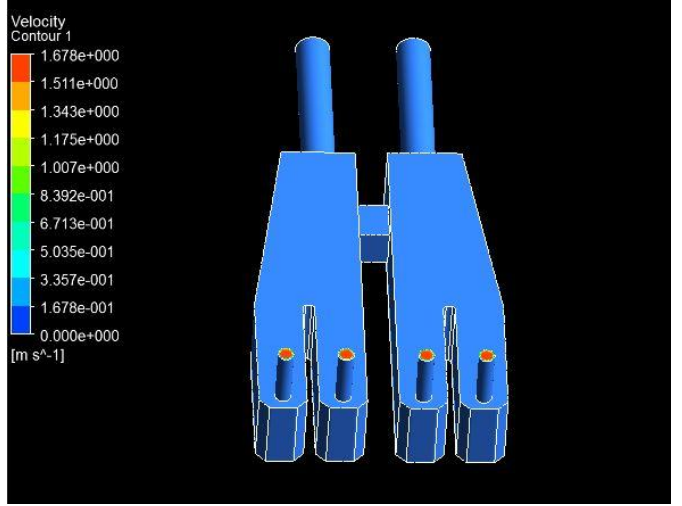

Fig.10 R1

Table 4- Comparative Study

\begin{tabular}{|c|c|c|c|c|}
\hline & \multicolumn{2}{|c|}{ SWIRL ANGLES (in Degrees) } & \multicolumn{2}{c|}{ VELOCITY (in m/s) at OUTLETS } \\
\cline { 1 - 2 } LOCATIONS & R0 & R1 & R0 & R1 \\
\hline TUBE 1 & 7.214 & 3.060 & \multirow{2}{*}{1.566} & \\
\hline TUBE 2 & 6.713 & 2.671 & & \\
\cline { 1 - 2 } TUBE 3 & 7.601 & 2.494 & & \\
\hline TUBE 4 & 6.243 & 3.026 & & \\
\hline
\end{tabular}

We can observe that swirl angles in all the tubes of model R0 were exceeding the limiting value i.e. 5 degrees [6] but in model R1 all are below limiting value. Also the velocity at the outlet has increased which implies that the flow at outlet has increased because of the smoother flow.

Further optimization was carried out by running CFD cases for different clearance values.

\section{BOTTOM SPLITTER AND GUIDE WALLS DESIGN:}

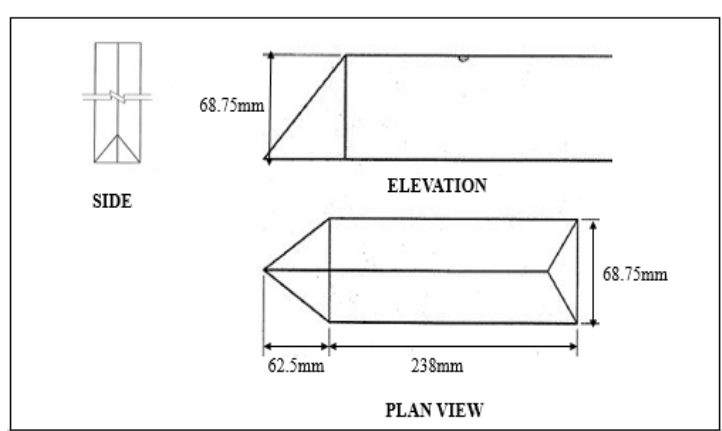

Fig.11 Bottom Splitter Dimensions

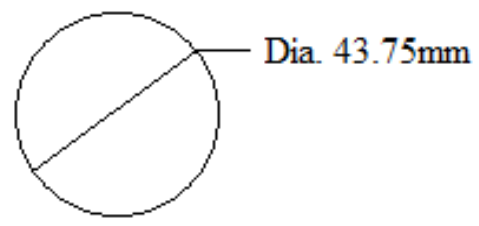

Guide walls are throught the height of sump

Fig.12 Guide Wall Dimensions

Minimization of swirl angle can be done by adding few attachments to the sump.

Bottom splitter and guide walls were designed with few recommended relations by HIS based on clearance value.

Above figures represent the different views and the dimensions of splitter and guide walls. 


\section{MODEL R5:}

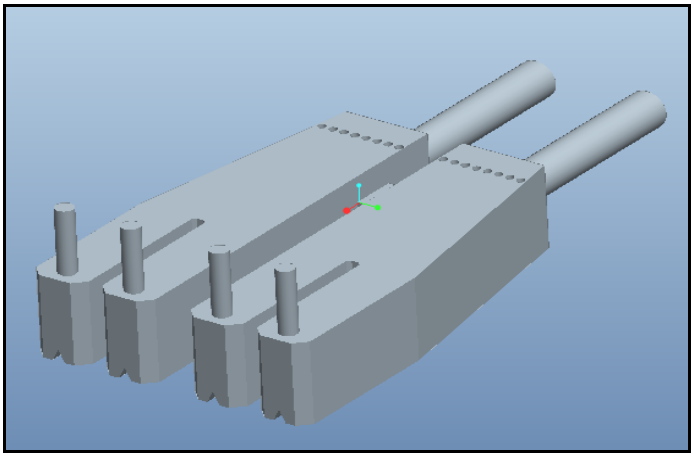

Fig.13: Solid Model R5

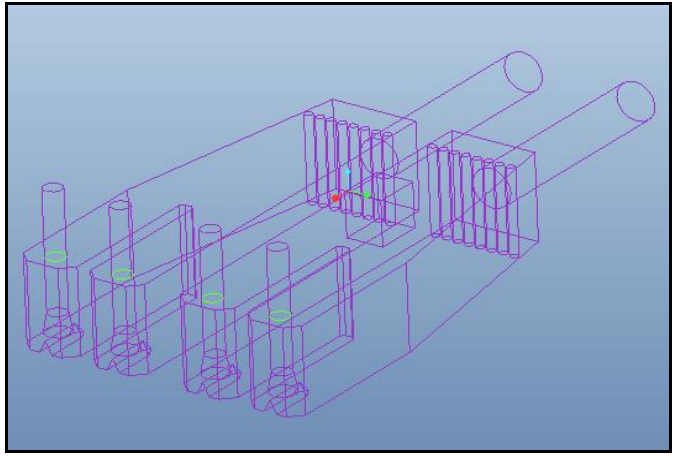

Fig.14:Wireframe Model R5

Modifications were done by adding bottom splitter and guide walls in the R1 and the CAD model was developed.

\section{STREAMLINE PLOTS:}

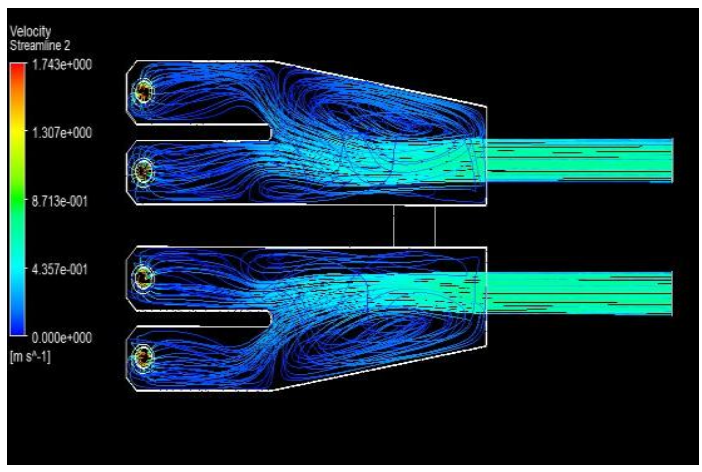

Fig.15:R0

VECTOR PLOTS:

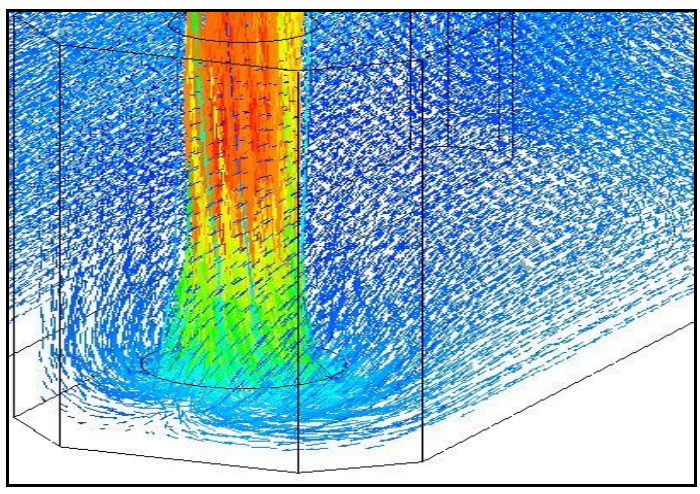

Fig.17:R0

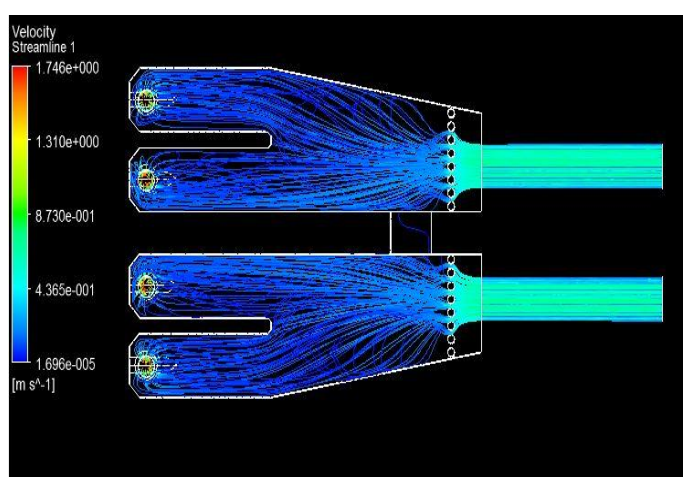

Fig.16: R1

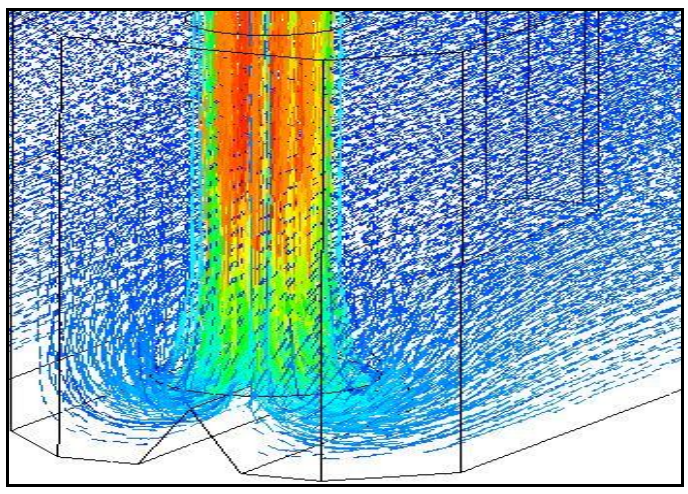

Fig.18:R1 
Table 5- Comparative Study

\begin{tabular}{|c|c|c|c|c|}
\hline & \multicolumn{2}{|c|}{ SWIRL ANGLES(in DEGREES) } & \multicolumn{2}{|c|}{ VELOCITY(in m/s) } \\
\cline { 1 - 3 } LOCATION & R1 & R5 & R1 & \multirow{2}{*}{1.678} \\
\cline { 1 - 2 } TUBE 1 & 3.060 & 0.670 & & 1.771 \\
\cline { 1 - 3 } TUBE 2 & 2.671 & 1.644 & \\
\cline { 1 - 2 } TUBE 3 & 2.494 & 1.796 & \\
\hline
\end{tabular}

\section{CONCLUSIONS}

1. Swirl angles are minimized with a significant amount and are well below the acceptable value.

2. Flow distribution is well and inter-circulations are absent.

3. Flow velocity at the outlet has increased up to $11.46 \%$ as compared to original model.

\section{REFERENCES}

[1] "Genaral Modelling and Scaling Laws", Experimental Methods in Marine Hydrodynamics, Chapter 2, NTNU.

[2] Samir A. S. Ibrahim, "Evaluation of the Flow Characteristics in the Intake Structure and Pump Sumps Using Physical Model”, Nile Basin Water Science \& Engineering Journal, Vol.4, Issue 1, 2011.

[3] Kadam Pratap M. \& D.S. Chavan, "CFD Analysis of Flow in Pump Sump to Check Suitability for Better Performance of Pump", ISSN, Volume-1, Issue-2, 2013.

[4] Tanweer S. Desmukh \& V.K Gahlot, "Simulation of Flow through A Pump Sump and its Validation", IJRRAS, July 2010.

[5] Tanweer S. Desmukh \& V.K Gahlot, "Numerical Study Of Flow Behaviour In A Multiple Intake Pump Sump", IJAET, Vol.2, Issue II, April-June, 2011.

[6] "American National Standard for Pump Intake Design”, Hydraulic Institute Standards, Nov 17, 1998.

[7] Chen Hong-xun, Guo Jia-hong, "Numerical Simulation Of 3-d Turbulent Flow in the Multiintakes Sump of the Pump Station", Science Direct Journal Of Hydrodynamics, China, March 2, 2006.

[8] Shazy A. Shabayek, "Improving Approach Flow Hydraulics at Pump Intakes", International Journal of Civil \& Environmental Engineering IJCEE-IJENS Vol: 10 No: 06, Dec 2010. 